\title{
On 2 - 3 Matrix Chevalley Eilenberg Cohomology
}

\author{
Joseph Dongho*, Epizitone Duebe-Abi, Shuntah Roland Yotcha \\ Department of Mathematics and Computer Science, Faculty of Sciences, University of Maroua, \\ Maroua, Cameroon \\ Email: "joseph.dongho@fs.univ-maroua.cm
}

Received 29 August 2015; accepted 13 December 2015; published 16 December 2015

Copyright (C) 2015 by authors and Scientific Research Publishing Inc.

This work is licensed under the Creative Commons Attribution International License (CC BY).

http://creativecommons.org/licenses/by/4.0/

c) (i) Open Access

\begin{abstract}
The main objective of this paper is to provide the tool rather than the classical adjoint representation of Lie algebra; which is essential in the conception of the Chevalley Eilenberg Cohomology. We introduce the notion of representation induced by a 2 - 3 matrix. We construct the corresponding Chevalley Eilenberg differential and we compute all its cohomological groups.
\end{abstract}

\section{Keywords}

Lie Algebra, Cochain, 2 - 3 Matrix Chevalley Eilenberg, Cohomology

\section{Introduction}

This work is included in the domain of differential geometry which is the continuation of infinitesimal calculation. It is possible to study it due to the new techniques of differential calculus and the new family of topological spaces applicable as manifold. The study of Lie algebra with classical example puts in place with so many homological materials [1]-[3] (Lie Bracket, Chevalley Eilenberg Cohomology...). The principal objective of this work is to introduce the notions of deformation of Lie algebra in the more general representation rather than the adjoint representation.

This work is base on 2 - 3 matrix Chevally Eilenberg Chohomology representation, in which our objective is to fixed a matrix representation and comes out with a representation which is different from the adjoint representation. Further, given a Lie algebra $V, W$ respectively of dimension 2 and 3, we construct a linear map that will define a Lie algebra structure from a Lie algebra $V$ into $E n d W$ by putting the commutator structure in place.

${ }^{*}$ Corresponding author. 
This does lead us to a fundamental condition of our 2 - 3 matrix Chevalley Eilenberg Cohomology. We compute explicitly all the associated cohomological groups.

\section{2 - 3 Matrix Representation Theorem}

We begin by choosing $V$ to be a 2-dimensional vector space and $W$ a 3-dimensional vector space, then we called our cohomology on a domain vector space $V$ and codomain $W$ a 2 - 3 matrix Chevalley Eilenberg Cohomology. In what follow, we denoted for all $i \in \mathbb{N}$ by $C^{i}(V, W)$ the space of $i$-multilinear skew symmetric map on $V$ with valor in $W$; we also denoted by $\left(e_{i}\right)_{i=1}^{2}$ and $\left(w_{i}\right)_{i=1}^{3}$ respectively the basis of $V$ and $W$. We also suppose that $\rho: V \rightarrow \operatorname{End}(W)$ is a representation of the Lie algebra $(V, \mu)$ where $\mu$ is the associated Lie structure.

\subsection{Description of Cochain Spaces}

Since element of $\mu_{i}$ of $C^{i}(V, W)$ skew symmetric, then for all $k \in\{1,2\}$, we have $\mu_{i}\left(e_{k}, e_{k}\right)=0$ [4]. Let $x \in V$ and $w \in W$, we have $x=C_{1} e_{1}+C_{2} e_{2}$ and $w=C_{1}^{1} w^{1}+C_{2}^{1} w^{2}+C_{3}^{1} w^{3}$, where $C_{1}, C_{2}, C_{1}^{1}, C_{2}^{1}, C_{3}^{1} \in \mathbb{K}$.

$\mu_{0} \in C^{0}(V, W)$ implies that $\mu_{0}=\left(w^{1}, w^{2}, w^{3}\right) . \mu_{1} \in C^{1}(V, W)$ iff $\mu_{1}: V \rightarrow W$ is a linear map.

Then,

$$
\begin{aligned}
\mu_{1}(x) & =\mu_{1}\left(C_{1} e_{1}+C_{2} e_{2}\right) \\
& =C_{1} \mu_{1}\left(e_{1}\right)+C_{2} \mu_{1}\left(e_{2}\right), \text { where } \mu_{1}\left(e_{i}\right)=C_{i}^{1} w^{1}+C_{i}^{2} w^{2}+C_{i}^{3} w^{3}, i=1,2 \\
& =C_{1} \sum_{i=1}^{3} C_{i}^{1} w^{i}+C_{2} \sum_{i=1}^{3} C_{i}^{2} w^{i}, \\
& =C^{1}\left(C_{1}^{1} w^{1}+C_{2}^{1} w^{2}+C_{3}^{1} w^{3}\right)+C^{2}\left(C_{1}^{2} w^{1}+C_{2}^{2} w^{2}+C_{3}^{2} w^{3}\right) .
\end{aligned}
$$

Lemma 1: If the $\operatorname{dim} V=2$ and $\operatorname{dim} W=3$, then $C^{1}(V, W) \cong M_{3 \times 2}(\mathbb{K})$.

Proof. Since $\mu_{1} \in C^{1}(V, W)$ then,

$$
\mu_{1}(x)=C^{1}\left(C_{1}^{1} w^{1}+C_{2}^{1} w^{2}+C_{3}^{1} w^{3}\right)+C^{2}\left(C_{1}^{2} w^{1}+C_{2}^{2} w^{2}+C_{3}^{2} w^{3}\right) .
$$

Thus, we define an isomorphic map $i_{1}$ from $C_{1}(V, W)$ to $M_{3 \times 2}(\mathbb{K})$ as follows;

$$
i_{1}\left(\mu_{1}(x)\right)=\left(\begin{array}{cc}
C_{1}^{1} & C_{1}^{2} \\
C_{2}^{1} & C_{2}^{2} \\
C_{3}^{1} & C_{3}^{2}
\end{array}\right) .
$$

$\mu_{2} \in C^{2}(V, W)$ iff $\mu_{2}: V \times V \rightarrow W$ is bilinear and antisymmetric map; then

$$
\begin{aligned}
\mu_{2}(x, y) & =\mu_{2}\left(C_{11} e_{1}+C_{12} e_{2}, C_{21} e_{1}+C_{22} e_{2}\right) \\
& =\mu_{2}\left(C_{11} e_{1}, C_{21} e_{1}\right)+\mu_{2}\left(C_{11} e_{1}, C_{22} e_{2}\right)+\mu_{2}\left(C_{12} e_{2}, C_{21} e_{1}\right)+\mu_{2}\left(C_{12} e_{2}, C_{22} e_{2}\right) \\
& =C_{11} C_{21} \mu_{2}\left(e_{1}, e_{1}\right)+C_{11} C_{22} \mu_{2}\left(e_{1}, e_{2}\right)+C_{12} C_{21} \mu_{2}\left(e_{2}, e_{1}\right)+C_{12} C_{22} \mu_{2}\left(e_{2}, e_{2}\right) \\
& =\left(C_{11} C_{22}-C_{12} C_{21}\right) \mu_{2}\left(e_{1}, e_{2}\right) \\
& =\left(C_{11} C_{22}-C_{12} C_{22}\right) \sum_{k=1}^{3} C_{12}^{k} w_{k} \\
& =\left(C_{11} C_{22}-C_{12} C_{22}\right)\left(C_{12}^{1} \omega^{1}+C_{12}^{2} \omega^{2}+C_{12}^{3} \omega^{3}\right) .
\end{aligned}
$$

Lemma 2: If $\operatorname{dim} V=2$ and $\operatorname{dim} W=3$ then $C^{2}(V, W) \cong M_{3 \times 1}(\mathbb{K})$.

Proof. From the expression of an element $\mu_{2}$ in $C^{2}(V, W)$ from above, $\mu_{2}$ can be represented as a column matrix $\left(\begin{array}{l}C_{12}^{1} \\ C_{12}^{2} \\ C_{12}^{3}\end{array}\right)$ of the lie constant structures. 

then

$\mu_{3} \in C^{3}(V, W)$ iff $\mu_{3}: V \times V \times V \rightarrow W$ is a tri-linear and skew symmetric map,

$$
\begin{aligned}
\mu_{3}(x, y, z)= & \mu_{3}\left(C_{11} e_{1}+C_{12} e_{2}, C_{21} e_{1}+C_{22} e_{2}, C_{31} e_{1}+C_{32} e_{2}\right) \\
= & \mu_{3}\left(C_{11} e_{1}, C_{21} e_{1}+C_{22} e_{2}, C_{31} e_{1}+C_{32} e_{2}\right)+\mu_{3}\left(C_{12} e_{2}, C_{21} e_{1}+C_{22} e_{2}, C_{31} e_{1}+C_{32} e_{2}\right) \\
= & \mu_{3}\left(C_{11} e_{1}, C_{21} e_{1}, C_{31} e_{1}+C_{32} e_{2}\right)+\mu_{3}\left(C_{11} e_{1}, C_{22} e_{2}, C_{31} e_{1}+C_{32} e_{2}\right) \\
& +\mu_{3}\left(C_{12} e_{2}, C_{21} e_{1}, C_{31} e_{1}+C_{32} e_{2}\right)+\mu_{3}\left(C_{12} e_{2}, C_{22} e_{2}, C_{31} e_{1}+C_{32} e_{2}\right) \\
= & \mu_{3}\left(C_{11} e_{1}, C_{21} e_{1}, C_{31} e_{1}\right)+\mu_{3}\left(C_{11} e_{1}, C_{21} e_{1}, C_{32} e_{2}\right)+\mu_{3}\left(C_{11} e_{1}, C_{22} e_{2}, C_{31} e_{1}\right) \\
& +\mu_{3}\left(C_{12} e_{2}, C_{21} e_{1}, C_{32} e_{2}\right)+\mu_{3}\left(C_{12} e_{2}, C_{20} e_{2}, C_{11} e_{1}\right)+\mu_{3}\left(C_{12} e_{2}, C_{22} e_{2}, C_{32} e_{2}\right) \\
& +\mu_{3}\left(C_{12} e_{2}, C_{21} e_{1}, C_{31} e_{1}\right)+\mu_{3}\left(C_{11} e_{1}, C_{22} e_{2}, C_{32} e_{2}\right) \\
= & C_{11} C_{21} C_{31} \mu_{3}\left(e_{1}, e_{1}, e_{1}\right)+C_{11} C_{21} C_{32}\left(e_{1}, e_{1}, e_{2}\right) \\
& +C_{11} C_{22} C_{31} \mu_{3}\left(e_{1}, e_{2}, e_{1}\right)+C_{11} C_{22} C_{32} \mu_{3}\left(e_{1}, e_{2}, e_{2}\right) \\
= & C_{12} C_{21} C_{31} \mu_{3}\left(e_{2}, e_{1}, e_{1}\right)+C_{12} C_{21} C_{32} \mu_{3}\left(e_{2}, e_{1}, e_{2}\right) \\
& +C_{12} C_{12} C_{31} \mu_{3}\left(e_{2}, e_{2}, e_{1}\right)+C_{12} C_{22} C_{32} \mu_{3}\left(e_{2}, e_{2}, e_{2}\right) \\
= & 0
\end{aligned}
$$

since $\mu_{3}$ is a linear anti-symmetric mapping.

Lemma 3: If $\operatorname{dim} V=2$ and $\operatorname{dim} W=3$ then $C^{3}(V, W) \cong 0$.

Proof. Since for every $\mu_{3} \in C^{3}(V, W)$, we have that $\mu_{3} \equiv 0$ from the expression of $\mu_{3}$ above.

\subsection{Diagram of a Sequence of Linear Maps}

According to the above results, we have the following diagram where we shall identify and define $d^{0}, d^{1}, d^{2}$ and $i_{0}, i_{1}, i_{2}$ in order to contruct our 2 - 3 Matrix Chevalley-Eilenberg Cohomology.

$$
\begin{array}{ccccccccc}
0 & \rightarrow & \underset{\text { \&2ll }}{\mathrm{W}} & \stackrel{d^{0}}{\rightarrow} & C^{1}(V, W) & \stackrel{d^{1}}{\rightarrow} & C^{2}(V, W) & \stackrel{d^{2}}{\rightarrow} & 0 \\
0 & \rightarrow & \mathrm{W} & \stackrel{\tilde{d}^{0}}{\rightarrow} & M_{3 \times 2}(\mathbb{R}) & \stackrel{\tilde{d}^{1}}{\rightarrow} & M_{3 \times 1}(\mathbb{R}) & \stackrel{\tilde{d}^{2}}{\rightarrow} & 0
\end{array}
$$

Expression of $d^{0}[1][4]$ :

$$
\begin{aligned}
d^{0}: W & \rightarrow C^{1}(V, W) \\
w & \mapsto d^{0} w: V \rightarrow W \\
v & \mapsto d^{0} w(v)=\rho(v) w
\end{aligned}
$$

Expression of $d^{1}[1][4]$ :

$$
\begin{aligned}
& d^{1}: C^{1}(V, W) \rightarrow C^{2}(V, W) \\
d^{1} \alpha(x, y)= & \delta \alpha(x, y), \text { for all } \alpha \text { in } C^{1}(V, W) \\
= & x \alpha(y)-y \alpha(x)-\alpha(\mu(x, y)) \\
= & \rho(x) \alpha(y)-\rho(y) \alpha(x)-\alpha(\mu(x, y))
\end{aligned}
$$

Expression of $d^{2}[1][4]$ :

$$
d^{2}: C(V, W) \rightarrow 0
$$

$$
\begin{aligned}
d^{2} \alpha(x, y, z)= & \delta \alpha(x, y, z), \text { for all } \alpha \text { in } C^{2}(V, W) \\
= & x \alpha(y, z)-y \alpha(z, x)+z \alpha(x, y)-\alpha(\mu(x, y), z) \\
& +\alpha(\mu(x, z), y)-\alpha(\mu(y, z), x) \\
\equiv & 0,
\end{aligned}
$$


since $d^{2}$ is mapped to the zero space. A direct computation, give us [1]

$$
d^{2} \circ d^{1}=0
$$

Definition of $i_{0}$ :

$$
\begin{gathered}
i_{0}: W \rightarrow W \\
i_{0}(w)=w, \forall w \in W
\end{gathered}
$$

i.e $i_{0}$ is the identity mappings from $W$ to $W$.

Definition of $i_{1}$ :

$$
\begin{gathered}
i_{1}: C^{1}(V, W) \rightarrow M_{3 \times 2}(\mathbb{R}) \\
i_{1}\left(\mu_{1}(x)\right)=\left(\begin{array}{ll}
C_{1}^{1} & C_{1}^{2} \\
C_{2}^{1} & C_{2}^{2} \\
C_{3}^{1} & C_{3}^{2}
\end{array}\right),
\end{gathered}
$$

which is the matrix of $\alpha_{1}, C_{j}^{i} \in \mathbb{R}, \quad i=1,2$ and $j=1,2,3$.

Definition of $i_{2}$ :

$$
\begin{gathered}
i_{2}: C^{2}(V, W) \rightarrow M_{3 \times 2}(\mathbb{R}) \\
i_{2}\left(\mu_{2}\left(e_{1}, e_{2}\right)\right)=\left(\begin{array}{l}
C_{12}^{1} \\
C_{12}^{2} \\
C_{12}^{3}
\end{array}\right),
\end{gathered}
$$

which is the matrix of $\alpha_{2}, C_{12}^{l} \in \mathbb{R}, \quad l=1,2,3$.

\subsection{Homological Differential}

In this section, we are going to determine expressions of $\tilde{d}^{1}, \tilde{d}^{0}$ and also prove that $d^{1} o d^{0}=0, \quad \tilde{d}^{1} \tilde{d}^{0}=0$ for us to obtain our 2 - 3 matrix Chevalley-Eilenberg differential complex. This is possible unless by stating an important hypothesis which we call 2 - 3 matrix Chevalley-Eilenberg hypothesis.

Proposition 1: If $\rho(x) \rho(y)-\rho(y) \rho(x)-\rho(\mu(x, y))=0$, for all $x, y$ in $V$, then $d^{1} o d^{0}=0$.

Proof. We assume that $\rho(x) \rho(y)-\rho(y) \rho(x)-\rho(\mu(x, y))=0$, for all $x, y$ in $V$.

By definition, we have that

$$
\begin{aligned}
& d^{0} w(x)=\rho(x) w \\
& d^{1} \alpha(x, y)=\rho(x) \alpha(y)-\rho(y) \alpha(x)-\alpha(\mu(x, y)) .
\end{aligned}
$$

Then by substituting equation (1) into (2),we have

$$
\begin{aligned}
d^{1} d^{0} w(x, y) & =\rho(x) d^{0} w(y)-\rho(y) d^{0} w(x)-d^{0} w(\mu(x, y)) \\
& =\rho(x) \rho(y) w-\rho(y) \rho(x) w-\rho(\mu(x, y)) w \\
& =[\rho(x) \rho(y)-\rho(y) \rho(x)-\rho(\mu(x, y))] w \\
& =0
\end{aligned}
$$

by hypothesis.

Expression of $\tilde{d}^{1}$ :

Let $V$ be a two dimensional Lie-algebra with basis $\left(e_{1}, e_{2}\right)$ and the Lie's bracket $\mu(x, y)$ where $\mu\left(e_{1}, e_{2}\right)=C_{12}^{1} e_{1}+C_{12}^{2} e_{2}$ and $W$ a three dimensional vector space with basis $\left(w^{1}, w^{2}, w^{3}\right)$. We define $\rho: V \rightarrow \operatorname{End}(W)$ by $v \mapsto \rho(v):=f_{M_{v}}$, where $M_{v}=\left(\begin{array}{lll}v_{11} & v_{12} & v_{13} \\ v_{21} & v_{22} & v_{23} \\ v_{31} & v_{32} & v_{33}\end{array}\right)$ and $f_{M_{v}}$ is a linear mapping associated to the matrix $M_{v}$. 
Let $M=\left(\begin{array}{ll}a_{1} & a_{2} \\ b_{1} & b_{2} \\ c_{1} & c_{2}\end{array}\right) \in M_{3 \times 2} \mapsto f_{M}: V \rightarrow W \quad$ defined by

$$
f_{M}\left(\begin{array}{l}
x \\
y
\end{array}\right)=\left(\begin{array}{l}
a_{1} x+a_{2} y \\
b_{1} x+b_{2} y \\
c_{1} x+c_{2} y
\end{array}\right):=M\left(\begin{array}{l}
x \\
y
\end{array}\right)
$$

Therefore;

$$
d^{1} f_{M}\left(\boldsymbol{v}_{1}, \boldsymbol{v}_{2}\right)=-\boldsymbol{v}_{1} \cdot f_{M}\left(\boldsymbol{v}_{2}\right)+\boldsymbol{v}_{2} \cdot f_{M}\left(\boldsymbol{v}_{1}\right)-f_{M}\left(\mu\left(\boldsymbol{v}_{1}, \boldsymbol{v}_{2}\right)\right)
$$

Since

$$
\begin{aligned}
\mu\left(\boldsymbol{v}_{1}, \boldsymbol{v}_{2}\right) & =\mu\left(v_{1}^{1} e_{1}+v_{2}^{1} e_{2}, v_{1}^{2} e_{1}+v_{2}^{2} e_{2}\right) \\
& =\mu\left(v_{1}^{1} e_{1}, v_{1}^{2} e_{2}\right)+\mu\left(v_{1}^{1} e_{1}, v_{2}^{2} e_{2}\right)+\mu\left(v_{2}^{1} e_{2}, v_{1}^{2} e_{1}\right)+\mu\left(v_{2}^{1} e_{2}, v_{2}^{2} e_{2}\right) \\
& =\left(v_{1}^{1} v_{2}^{2}-v_{2}^{1} v_{1}^{2}\right) \mu\left(e_{1}, e_{2}\right)=\left(v_{1}^{1} v_{2}^{2}-v_{2}^{1} v_{1}^{2}\right)\left(C_{12}^{1} e_{1}+C_{12}^{2} e_{2}\right) \\
& =C_{12}^{1}\left(v_{1}^{1} v_{2}^{2}-v_{2}^{1} v_{1}^{2}\right) e_{1}+C_{12}^{2}\left(v_{1}^{1} v_{2}^{2}-v_{2}^{1} v_{1}^{2}\right) e_{2} .
\end{aligned}
$$

Therefore,

$$
\begin{aligned}
f_{M}\left(\mu\left(\boldsymbol{v}_{1}, \boldsymbol{v}_{2}\right)\right)= & f_{M}\left[\left(C_{12}^{1}\left(v_{1}^{1} v_{2}^{2}-v_{2}^{1} v_{1}^{2}\right) e_{1}+C_{12}^{2}\left(v_{1}^{1} v_{2}^{2}-v_{2}^{1} v_{1}^{2}\right) e_{2}\right)\right] \\
= & \left(a_{1} C_{12}^{1}\left(v_{1}^{1} v_{2}^{2}-v_{2}^{1} v_{1}^{2}\right)+a_{2} C_{12}^{2}\left(v_{1}^{1} v_{2}^{2}-v_{2}^{1} v_{1}^{2}\right)\right) \boldsymbol{w}_{1} \\
& +\left(b_{1} C_{12}^{1}\left(v_{1}^{1} v_{2}^{2}-v_{2}^{1} v_{1}^{2}\right)+b_{2} C_{12}^{2}\left(v_{1}^{1} v_{2}^{2}-v_{2}^{1} v_{1}^{2}\right)\right) \boldsymbol{w}_{2} \\
& +\left(c_{1} C_{12}^{1}\left(v_{1}^{1} v_{2}^{2}-v_{2}^{1} v_{1}^{2}\right)+c_{2} C_{12}^{2}\left(v_{1}^{1} v_{2}^{2}-v_{2}^{1} v_{1}^{2}\right)\right) \boldsymbol{w}_{3} .
\end{aligned}
$$

Also, we have

$$
f_{M}\left(v_{1}\right)=\left(\begin{array}{l}
a_{1} v_{1}^{1}+a_{2} v_{2}^{1} \\
b_{1} v_{1}^{1}+b_{2} v_{2}^{1} \\
c_{1} v_{1}^{1}+c_{2} v_{2}^{1}
\end{array}\right), \quad f_{M}\left(v_{2}\right)=\left(\begin{array}{l}
a_{1} v_{1}^{2}+a_{2} v_{2}^{2} \\
b_{1} v_{1}^{2}+b_{2} v_{2}^{2} \\
c_{1} v_{1}^{2}+c_{2} v_{2}^{2}
\end{array}\right) .
$$

$\rho\left(v_{2}\right) \cdot f_{M}\left(v_{1}\right)=M_{v_{2}} \cdot f_{M}\left(v_{1}\right)$, where

$$
M_{v_{2}}=\left(\begin{array}{ccc}
v_{11}^{2} & v_{12}^{2} & v_{13}^{2} \\
v_{21}^{2} & v_{22}^{2} & v_{23}^{2} \\
v_{31}^{2} & v_{32}^{2} & v_{33}^{2}
\end{array}\right)
$$

$\rho\left(v_{1}\right) \cdot f_{M}\left(v_{2}\right)$, where

$$
M_{v_{1}}=\left(\begin{array}{ccc}
v_{11}^{1} & v_{12}^{1} & v_{13}^{1} \\
v_{21}^{1} & v_{22}^{1} & v_{23}^{1} \\
v_{31}^{1} & v_{32}^{1} & v_{33}^{1}
\end{array}\right)
$$

So,

$$
\begin{aligned}
M_{v_{2}} \cdot f_{M}\left(\boldsymbol{v}_{1}\right)= & \left(\left(a_{1} v_{1}^{1}+a_{2} v_{2}^{1}\right) v_{11}^{2}+\left(b_{1} v_{1}^{1}+b_{2} v_{2}^{1}\right) v_{21}^{2}+\left(c_{1} v_{1}^{1}+c_{2} v_{2}^{1}\right) v_{31}^{2}\right) \boldsymbol{w}_{1} \\
& +\left(\left(a_{1} v_{1}^{1}+a_{2} v_{2}^{1}\right) v_{12}^{2}+\left(b_{1} v_{1}^{1}+b_{2} v_{2}^{1}\right) v_{22}^{2}+\left(c_{1} v_{1}^{1}+c_{2} v_{2}^{1}\right) v_{32}^{2}\right) \boldsymbol{w}_{2} \\
& +\left(\left(a_{1} v_{1}^{1}+a_{2} v_{2}^{1}\right) v_{13}^{2}+\left(b_{1} v_{1}^{1}+b_{2} v_{2}^{1}\right) v_{23}^{2}+\left(c_{1} v_{1}^{1}+c_{2} v_{2}^{1}\right) v_{33}^{2}\right) \boldsymbol{w}_{3} .
\end{aligned}
$$




$$
\begin{aligned}
M_{v_{1}} \cdot f_{M}\left(\boldsymbol{v}_{2}\right)= & \left(\left(a_{1} v_{1}^{2}+a_{2} v_{2}^{2}\right) v_{11}^{1}+\left(b_{1} v_{1}^{2}+b_{2} v_{2}^{2}\right) v_{21}^{1}+\left(c_{1} v_{1}^{2}+c_{2} v_{2}^{2}\right) v_{31}^{1}\right) \boldsymbol{w}_{1} \\
& +\left(\left(a_{1} v_{1}^{2}+a_{2} v_{2}^{2}\right) v_{12}^{1}+\left(b_{1} v_{1}^{2}+b_{2} v_{2}^{2}\right) v_{22}^{1}+\left(c_{1} v_{1}^{2}+c_{2} v_{2}^{2}\right) v_{32}^{1}\right) \boldsymbol{w}_{2} \\
& +\left(\left(a_{1} v_{1}^{2}+a_{2} v_{2}^{2}\right) v_{13}^{1}+\left(b_{1} v_{1}^{2}+b_{2} v_{2}^{2}\right) v_{23}^{1}+\left(c_{1} v_{1}^{2}+c_{2} v_{2}^{2}\right) v_{33}^{1}\right) \boldsymbol{w}_{3} .
\end{aligned}
$$

Therefore,

$$
\begin{aligned}
d^{1} & f_{M}\left(\boldsymbol{v}_{1}, v_{2}\right) \\
= & -M_{v_{1}} \cdot f_{M}\left(\boldsymbol{v}_{2}\right)+M_{v_{2}} \cdot f_{M}\left(\boldsymbol{v}_{1}\right)-f_{M}\left(\mu\left(\boldsymbol{v}_{1}, \boldsymbol{v}_{2}\right)\right) \\
= & {\left[\left(a_{1} v_{1}^{1}+a_{2} v_{2}^{1}\right) v_{11}^{2}-\left(a_{1} v_{1}^{2}+a_{2} v_{2}^{2}\right) v_{11}^{1}+\left(b_{1} v_{1}^{1}+b_{2} v_{2}^{1}\right) v_{21}^{2}-\left(b_{1} v_{1}^{2}+b_{2} v_{2}^{2}\right) v_{21}^{1}\right.} \\
& \left.+\left(c_{1} v_{1}^{1}+c_{2} v_{2}^{1}\right) v_{31}^{2}-\left(c_{1} v_{1}^{2}+c_{2} v_{2}^{2}\right) v_{31}^{1}-a_{1} C_{12}^{1}\left(v_{1}^{1} v_{2}^{2}-v_{2}^{1} v_{1}^{2}\right)-a_{2} C_{12}^{2}\left(v_{1}^{1} v_{2}^{2}-v_{2}^{1} v_{1}^{2}\right)\right] \boldsymbol{w}_{1} \\
& +\left[\left(a_{1} v_{1}^{1}+a_{2} v_{2}^{1}\right)-\left(a_{1} v_{1}^{2}+a_{2} v_{2}^{2}\right) v_{12}^{1}+\left(b_{1} v_{1}^{1}+b_{2} v_{2}^{1}\right) v_{22}^{2}-\left(b_{1} v_{1}^{2}+b_{2} v_{2}^{2}\right) v_{22}^{1}\right. \\
& \left.-\left(c_{1} v_{1}^{1}+c_{1} v_{2}^{1}\right) v_{32}^{2}-\left(c_{1} v_{1}^{2}+c_{2} v_{2}^{2}\right) v_{32}^{1}-b_{1} C_{12}^{1}\left(v_{1}^{1} v_{2}^{2}-v_{2}^{1} v_{1}^{2}\right)-b_{2} C_{12}^{1}\left(v_{1}^{1} v_{2}^{2}-v_{2}^{1} v_{1}^{2}\right)\right] \boldsymbol{w}_{2} \\
& +\left[\left(a_{1} v_{1}^{1}+a_{2} v_{2}^{1}\right) v_{13}^{2}-\left(a_{1} v_{1}^{2}+a_{2} v_{2}^{2}\right) v_{13}^{1}+\left(b_{1} v_{1}^{1}+b_{2} v_{2}^{1}\right) v_{23}^{2}-\left(b_{1} v_{1}^{2}+b_{2} v_{2}^{2}\right) v_{23}^{1}\right. \\
& \left.+\left(c_{1} v_{1}^{1}+c_{2} v_{2}^{1}\right) v_{33}^{2}-\left(c_{1} v_{1}^{2}+c_{2} v_{2}^{2}\right) v_{33}^{1}-c_{1} C_{12}^{1}\left(v_{1}^{1} v_{2}^{2}-v_{2}^{1} v_{1}^{2}\right)-c_{2} C_{12}^{2}\left(v_{1}^{1} v_{2}^{2}-v_{2}^{1} v_{1}^{2}\right)\right] \boldsymbol{w}_{3} .
\end{aligned}
$$

Now, we compute $d^{1} f_{M}\left(e_{1}, e_{2}\right)$ where $e_{1}$ and $e_{2}$ are basis vectors of $V$.

By replacing the constants $v_{1}^{1}=1, v_{2}^{1}=0, v_{1}^{2}=0$ and $v_{2}^{2}=1$, we obtain $d^{1} f_{M}\left(e_{1}, e_{2}\right)$ which is given as;

$$
\begin{aligned}
d^{1} f_{M}\left(e_{1}, e_{2}\right)= & \left(a_{1} e_{11}^{2}-a_{2} e_{11}^{1}+b_{1} e_{21}^{2}-b_{2} e_{21}^{1}+c_{1} e_{31}^{2}-c_{2} e_{31}^{1}-a_{1} C_{12}^{1}-a_{2} C_{12}^{2}\right) \boldsymbol{w}_{1} \\
& +\left(a_{1} e_{12}^{2}-a_{2} e_{12}^{1}+b_{1} e_{22}^{2}-b_{2} e_{22}^{1}+c_{1} e_{32}^{2}-c_{2} e_{32}^{1}-b_{1} C_{12}^{1}-b_{2} C_{12}^{1}\right) \boldsymbol{w}_{2} \\
& +\left(a_{1} e_{13}^{2}-a_{2} e_{13}^{1}+b_{1} e_{23}^{2}-b_{2} e_{23}^{1}+c_{1} e_{33}^{2}-c_{2} e_{33}^{1}-c_{1} C_{12}^{1}-c_{2} C_{12}^{2}\right) \boldsymbol{w}_{3} .
\end{aligned}
$$

Thus,

$$
\tilde{d}^{1} f_{M}\left(e_{1}, e_{2}\right)=\left(\begin{array}{l}
a_{1} e_{11}^{2}-a_{2} e_{11}^{1}+b_{1} e_{21}^{2}-b_{2} e_{21}^{1}+c_{1} e_{31}^{2}-c_{2} e_{31}^{1}-a_{1} C_{12}^{1}-a_{2} C_{12}^{2} \\
a_{1} e_{12}^{2}-a_{2} e_{12}^{1}+b_{1} e_{22}^{2}-b_{2} e_{22}^{1}+c_{1} e_{32}^{2}-c_{2} e_{32}^{1}-b_{1} C_{12}^{1}-b_{2} C_{12}^{2} \\
a_{1} e_{13}^{2}-a_{2} e_{13}^{1}+b_{1} e_{23}^{2}-b_{2} e_{23}^{1}+c_{1} e_{33}^{2}-c_{2} e_{33}^{1}-c_{1} C_{12}^{1}-c_{2} C_{12}^{2}
\end{array}\right) .
$$

Hence, $\tilde{d}^{1}: M_{3 \times 2} \rightarrow M_{3 \times 1}$ is defined by

$$
\begin{gathered}
\left(\begin{array}{ll}
a_{1} & a_{2} \\
b_{1} & b_{2} \\
c_{1} & c_{2}
\end{array}\right) \mapsto\left(\begin{array}{l}
a_{1} e_{11}^{2}-a_{2} e_{11}^{1}+b_{1} e_{21}^{2}-b_{2} e_{21}^{1}+c_{1} e_{31}^{2}-c_{2} e_{31}^{1}-a_{1} C_{12}^{1}-a_{2} C_{12}^{2} \\
a_{1} e_{12}^{2}-a_{2} e_{12}^{1}+b_{1} e_{22}^{2}-b_{2} e_{22}^{1}+c_{1} e_{32}^{2}-c_{2} e_{32}^{1}-b_{1} C_{12}^{1}-b_{2} C_{12}^{2} \\
a_{1} e_{13}^{2}-a_{2} e_{13}^{1}+b_{1} e_{23}^{2}-b_{2} e_{23}^{1}+c_{1} e_{33}^{2}-c_{2} e_{33}^{1}-c_{1} C_{12}^{1}-c_{2} C_{12}^{2}
\end{array}\right) \text { with } \\
M_{e_{1}}=\left(\begin{array}{lll}
e_{11}^{1} & e_{12}^{1} & e_{13}^{1} \\
e_{21}^{1} & e_{22}^{1} & e_{23}^{1} \\
e_{31}^{1} & e_{32}^{1} & e_{33}^{1}
\end{array}\right), M_{e_{2}}=\left(\begin{array}{lll}
e_{11}^{2} & e_{12}^{2} & e_{13}^{2} \\
e_{21}^{2} & e_{22}^{2} & e_{23}^{2} \\
e_{31}^{2} & e_{32}^{2} & e_{33}^{2}
\end{array}\right) .
\end{gathered}
$$

Corollary 1: If

$$
\begin{aligned}
& d^{1} f_{M}\left(e_{1}, e_{2}\right) \\
= & \left(a_{1} e_{11}^{2}-a_{2} e_{11}^{1}+b_{1} e_{21}^{2}-b_{2} e_{21}^{1}+c_{1} e_{31}^{2}-c_{2} e_{31}^{1}-a_{1} C_{12}^{1}-a_{2} C_{12}^{2}\right) \boldsymbol{w}_{1} \\
& +\left(a_{1} e_{12}^{2}-a_{2} e_{12}^{1}+b_{1} e_{22}^{2}-b_{2} e_{22}^{1}+c_{1} e_{32}^{2}-c_{2} e_{32}^{1}-b_{1} C_{12}^{1}-b_{2} C_{12}^{1}\right) \boldsymbol{w}_{2} \\
& +\left(a_{1} e_{13}^{2}-a_{2} e_{13}^{1}+b_{1} e_{23}^{2}-b_{2} e_{23}^{1}+c_{1} e_{33}^{2}-c_{2} e_{33}^{1}-c_{1} C_{12}^{1}-c_{2} C_{12}^{2}\right) \boldsymbol{w}_{3}
\end{aligned}
$$

then $\tilde{d}^{1}: M_{3 \times 2} \rightarrow M_{3 \times 1}$ is defined by 


$$
\left(\begin{array}{ll}
a_{1} & a_{2} \\
b_{1} & b_{2} \\
c_{1} & c_{2}
\end{array}\right) \mapsto\left(\begin{array}{l}
a_{1} e_{11}^{2}-a_{2} e_{11}^{1}+b_{1} e_{21}^{2}-b_{2} e_{21}^{1}+c_{1} e_{31}^{2}-c_{2} e_{31}^{1}-a_{1} C_{12}^{1}-a_{2} C_{12}^{2} \\
a_{1} e_{12}^{2}-a_{2} e_{12}^{1}+b_{1} e_{22}^{2}-b_{2} e_{22}^{1}+c_{1} e_{32}^{2}-c_{2} e_{32}^{1}-b_{1} C_{12}^{1}-b_{2} C_{12}^{2} \\
a_{1} e_{13}^{2}-a_{2} e_{13}^{1}+b_{1} e_{23}^{2}-b_{2} e_{23}^{1}+c_{1} e_{33}^{2}-c_{2} e_{33}^{1}-c_{1} C_{12}^{1}-c_{2} C_{12}^{2}
\end{array}\right)
$$

\subsection{Fundametal Condition of 2 - 3 Matrix Chevalley-Eilenberg Cohomology}

We now state the main hypothesis for our 2 - 3 matrix Chevalley-Eilenberg Cohomology, which we suppose that $\rho\left(e_{1}\right) \rho\left(\left(e_{2}\right)-\rho\left(e_{2}\right) \rho\left(e_{1}\right)-\rho\left(\mu\left(e_{1}, e_{2}\right)\right)=0\right.$.

$$
\begin{aligned}
& \text { i.e } M_{e_{1}} M_{e_{2}}-M_{e_{2}} M_{e_{1}}-C^{1} M_{e_{1}}-C_{12}^{2} M_{e_{2}}=0 \\
& \quad\left[\left(e_{11}^{1} e_{11}^{2}+e_{12}^{1} e_{21}^{2}+e_{13}^{1} e_{31}^{2}\right)-\left(e_{11}^{2} e_{11}^{1}+e_{12}^{2} e_{21}^{1}+e_{13}^{2} e_{31}^{1}\right)+\left(e_{11}^{1} e_{12}^{2}+e_{12}^{1} e_{22}^{2}+e_{13}^{1} e_{32}^{2}\right)\right. \\
& \text { i.e } \quad-\left(e_{11}^{2} e_{12}^{1}+e_{12}^{2} e_{22}^{1}+e_{13}^{2} e_{32}^{1}\right)+\left(e_{11}^{1} e_{13}^{2}+e_{12}^{1} e_{23}^{2}+e_{13}^{1} e_{33}^{2}\right)-\left(e_{11}^{2} e_{13}^{1}+e_{12}^{2} e_{23}^{1}+e_{13}^{2} e_{33}^{1}\right), \\
& \left.\quad-C_{12}^{1} e_{11}^{1}-C_{12}^{2} e_{11}^{2}-C_{12}^{1} e_{12}^{1}-C_{12}^{2} e_{12}^{2}-C_{12}^{1} e_{13}^{1}-C_{12}^{2} e_{13}^{2}\right]=0 \\
& {\left[\left(e_{21}^{1} e_{11}^{2}+e_{22}^{1} e_{21}^{2}+e_{23}^{1} e_{31}^{2}\right)-\left(e_{21}^{2} e_{11}^{1}+e_{22}^{2} e_{21}^{1}+e_{23}^{2} e_{31}^{1}\right)+\left(e_{21}^{1} e_{12}^{2}+e_{22}^{1} e_{22}^{2}+e_{23}^{1} e_{32}^{2}\right)\right.} \\
& -\left(e_{21}^{2} e_{12}^{1}+e_{22}^{2} e_{22}^{1}+e_{23}^{2} e_{32}^{1}\right)+\left(e_{21}^{1} e_{13}^{2}+e_{22}^{1} e_{23}^{2}+e_{23}^{1} e_{33}^{2}\right)-\left(e_{21}^{2} e_{13}^{1}+e_{22}^{2} e_{23}^{1}+e_{23}^{2} e_{33}^{1}\right) \text { and } \\
& \left.-C_{12}^{1} e_{21}^{1}-C_{12}^{2} e_{21}^{2}-C_{12}^{1} e_{22}^{1}-C_{12}^{2} e_{22}^{2}-C_{12}^{1} e_{23}^{1}-C_{12}^{2} e_{23}^{2}\right]=0 \\
& {\left[\left(e_{31}^{1} e_{11}^{2}+e_{32}^{1} e_{21}^{2}+e_{33}^{1} e_{31}^{2}\right)-\left(e_{31}^{2} e_{11}^{1}+e_{32}^{2} e_{21}^{1}+e_{33}^{2} e_{31}^{1}\right)+\left(e_{31}^{1} e_{12}^{2}+e_{32}^{1} e_{22}^{2}+e_{33}^{1} e_{32}^{2}\right)\right.} \\
& -\left(e_{31}^{2} e_{12}^{1}+e_{32}^{2} e_{22}^{1}+e_{33}^{2} e_{32}^{1}\right)+\left(e_{31}^{1} e_{13}^{2}+e_{32}^{1} e_{23}^{2}+e_{33}^{1} e_{33}^{2}\right)-\left(e_{31}^{2} e_{13}^{1}+e_{32}^{2} e_{23}^{1}+e_{33}^{2} e_{33}^{1}\right) . \\
& \left.-C_{12}^{1} e_{31}^{1}-C_{12}^{2} e_{31}^{2}-C_{12}^{1} e_{32}^{1}-C_{12}^{2} e_{32}^{2}-C_{12}^{1} e_{33}^{1}-C_{12}^{2} e_{33}^{2}\right]=0
\end{aligned}
$$

This is an important tool in the construction of our 2 - 3 matrix cohomology differential complex.

\subsection{Expression of $\tilde{d}^{0}$}

From the diagram,

$$
\begin{aligned}
& \tilde{d}^{0}=i_{1} \circ d^{0} \circ i_{0}^{-1} \\
& i_{0}: W \rightarrow W \\
& w \mapsto i(w)=1_{w}(w)=w \quad \forall w \in W . \\
& d^{0}: W \rightarrow C^{1}(V, W) \\
& w \mapsto \rho(w) v=f_{M_{v}}:=\left(\begin{array}{lll}
v_{11} & v_{12} & v_{13} \\
v_{21} & v_{22} & v_{23} \\
v_{31} & v_{32} & v_{33}
\end{array}\right)
\end{aligned}
$$

where

$$
\begin{aligned}
d^{0} M(v) & =\left(\begin{array}{lll}
v_{11} & v_{12} & v_{13} \\
v_{21} & v_{22} & v_{23} \\
v_{31} & v_{32} & v_{33}
\end{array}\right)\left(\begin{array}{c}
w^{1} \\
w^{2} \\
w^{3}
\end{array}\right) \\
& =\left(\begin{array}{l}
v_{11} w^{1}+v_{12} w^{2}+v_{13} w^{3} \\
v_{21} w^{1}+v_{22} w^{2}+v_{23} w^{3} \\
v_{31} w^{1}+v_{32} w^{2}+v_{33} w^{3}
\end{array}\right)
\end{aligned}
$$




$$
\begin{aligned}
{\left[\left(\tilde{d}^{0}(M)\left(e_{1}\right) \tilde{d}^{0}(M)\left(e_{2}\right)\right)\right] } & =\left(\left(\begin{array}{lll}
e_{11}^{1} & e_{12}^{1} & e_{13}^{1} \\
e_{21}^{1} & e_{22}^{1} & e_{23}^{1} \\
e_{31}^{1} & e_{32}^{1} & e_{33}^{1}
\end{array}\right)\left(\begin{array}{l}
w^{1} \\
w^{2} \\
w^{3}
\end{array}\right)\left(\begin{array}{lll}
e_{11}^{2} & e_{12}^{2} & e_{13}^{2} \\
e_{21}^{2} & e_{22}^{2} & e_{23}^{2} \\
e_{31}^{2} & e_{32}^{2} & e_{33}^{2}
\end{array}\right)\left(\begin{array}{c}
w^{1} \\
w^{2} \\
w^{3}
\end{array}\right)\right) \\
& =\left(\begin{array}{cc}
e_{11}^{1} w^{1}+e_{12}^{1} w^{2}+e_{13}^{1} w^{3} & e_{11}^{2} w^{1}+e_{12}^{2} w^{2}+e_{13}^{2} w^{3} \\
e_{21}^{1} w^{1}+e_{22}^{1} w^{2}+e_{23}^{1} w^{3} & e_{21}^{2} w^{1}+e_{22}^{2} w^{2}+e_{23}^{2} w^{3} \\
e_{31}^{1} w^{1}+e_{32}^{1} w^{2}+e_{33}^{1} w^{3} & e_{31}^{2} w^{1}+e_{32}^{2} w^{2}+e_{33}^{2} w^{3}
\end{array}\right)
\end{aligned}
$$

$\boldsymbol{v}=\left(\begin{array}{l}x \\ y\end{array}\right)$ and $W=\left\langle w_{1}, w_{2}, w_{3}\right\rangle$. Thus, using the basis vectors $e_{1}$ and $e_{2}$ in $V$, we have

$$
\tilde{d}^{0}\left(e_{1}, e_{2}\right)=\left(\begin{array}{cc}
e_{11}^{1} w^{1}+e_{12}^{1} w^{2}+e_{13}^{1} w^{3} & e_{11}^{2} w_{1}+e_{12}^{2} w^{2}+e_{13}^{2} w^{3} \\
e_{21}^{1} w^{1}+e_{22}^{1} w^{2}+e_{23}^{1} w^{3} & e_{21}^{2} w^{1}+e_{22}^{2} w^{2}+e_{23}^{2} w^{3} \\
e_{31}^{1} w^{1}+e_{32}^{1} w^{2}+e_{33}^{1} w^{3} & e_{31}^{2} w^{1}+e_{32}^{2} w^{2}+e_{33}^{2} w^{3}
\end{array}\right)=\left(\begin{array}{cc}
\gamma_{1} & \gamma_{2} \\
\gamma_{3} & \gamma_{4} \\
\gamma_{5} & \gamma_{6}
\end{array}\right)
$$

Hence, the mapping $\tilde{d}^{0}: W \rightarrow M_{3 \times 2}$ is defined as;

$$
\left(\begin{array}{l}
w_{1} \\
w_{2} \\
w_{3}
\end{array}\right) \mapsto\left(\begin{array}{ll}
e_{11}^{1} w^{1}+e_{12}^{1} w^{2}+e_{13}^{1} w^{3} & e_{11}^{2} w^{1}+e_{12}^{2} w^{2}+e_{13}^{2} w^{3} \\
e_{21}^{1} w^{1}+e_{22}^{1} w^{2}+e_{23}^{1} w^{3} & e_{21}^{2} w^{1}+e_{22}^{2} w^{2}+e_{23}^{2} w^{3} \\
e_{31}^{1} w^{1}+e_{32}^{1} w^{2}+e_{33}^{1} w^{3} & e_{31}^{2} w^{1}+e_{32}^{2} w^{2}+e_{33}^{2} w^{3}
\end{array}\right)
$$

Corollary 2: If $\tilde{d}^{0}\left(e_{1}, e_{2}\right)=\left(\begin{array}{ll}e_{11}^{1} w^{1}+e_{12}^{1} w^{2}+e_{13}^{1} w^{3} & e_{11}^{2} w_{1}+e_{12}^{2} w^{2}+e_{13}^{2} w^{3} \\ e_{21}^{1} w^{1}+e_{22}^{1} w^{2}+e_{23}^{1} w^{3} & e_{21}^{2} w^{1}+e_{22}^{2} w^{2}+e_{23}^{2} w^{3} \\ e_{31}^{1} w^{1}+e_{32}^{1} w^{2}+e_{33}^{1} w^{3} & e_{31}^{2} w^{1}+e_{32}^{2} w^{2}+e_{33}^{2} w^{3}\end{array}\right)$

then the mapping $\tilde{d}^{0}: W \rightarrow M_{3 \times 2}$ is defined as;

$$
\left(\begin{array}{l}
w_{1} \\
w_{2} \\
w_{3}
\end{array}\right) \mapsto\left(\begin{array}{ll}
e_{11}^{1} w^{1}+e_{12}^{1} w^{2}+e_{13}^{1} w^{3} & e_{11}^{2} w^{1}+e_{12}^{2} w^{2}+e_{13}^{2} w^{3} \\
e_{21}^{1} w^{1}+e_{22}^{1} w^{2}+e_{23}^{1} w^{3} & e_{21}^{2} w^{1}+e_{22}^{2} w^{2}+e_{23}^{2} w^{3} \\
e_{31}^{1} w^{1}+e_{32}^{1} w^{2}+e_{33}^{1} w^{3} & e_{31}^{2} w^{1}+e_{32}^{2} w^{2}+e_{33}^{2} w^{3}
\end{array}\right)
$$

The matrix $\tilde{d}^{0}\left(e_{1}, e_{2}\right)$ has been assigned to the matrix $\left(\begin{array}{ll}\gamma_{1} & \gamma_{2} \\ \gamma_{3} & \gamma_{4} \\ \gamma_{5} & \gamma_{6}\end{array}\right)$ to simplify the composition of $\tilde{d}^{1}$ and $\tilde{d}^{2}$.

Proposition 2: $\tilde{d}^{1} 0 \tilde{d}^{0}=0$.

Proof. Since $d^{2} \circ d^{1}=0, \tilde{d}^{1}=i_{1} \circ d^{1} o i_{1}^{-1}$ and $\tilde{d}^{0}=i_{1} o d^{0} o i_{0}^{-1}$, We have:

$$
\tilde{d}^{1} o \tilde{d}^{0}=\left(i_{1} o d^{1} o i_{1}^{-1}\right) o\left(i_{1} o d^{0} o i_{0}^{-1}\right)=i_{2} o d^{1} o\left(i_{1}^{-1} o i_{1}\right) o d^{0} o i_{0}^{-1}=i_{2} o d^{1} o d^{0} o i_{0}^{-1}=i_{2} 0 i_{0}^{-1}=0,
$$

Which gives us our 2 - 3 matrix Chevalley Eilenberg homological hypothesis

$$
\left(M_{e_{1}} M_{e_{2}}-M_{e_{2}} M_{e_{1}}-C_{12}^{1} M_{e_{1}}-C_{12}^{2} M_{e_{2}}=0\right) .
$$

Remark 1: By straightforward computation, we have

$$
\tilde{d}^{1} o \tilde{d}^{0}(M)=\left(\begin{array}{c}
\gamma_{1} e_{11}^{2} w^{1}-\gamma_{2} e_{11}^{1} w^{1}+\gamma_{3} e_{21}^{2} w^{1}-\gamma_{4} e_{21}^{1} w^{1}+\gamma_{5} e_{31}^{2} w^{1}-\gamma_{6} e_{31}^{1} w^{1}-\gamma_{1} C_{12}^{1} w^{1}-\gamma_{2} C_{12}^{2} w^{1} \\
\gamma_{1} e_{12}^{2} w^{2}-\gamma_{2} e_{12}^{1} w^{2}+\gamma_{3} e_{22}^{2} w^{2}-\gamma_{4} e_{22}^{1} w^{2}+\gamma_{5} e_{32}^{2} w^{2}-\gamma_{6} e_{32}^{1} w^{2}-\gamma_{3} C_{12}^{1} w^{2}-\gamma_{4} C_{12}^{2} w^{2} \\
\gamma_{1} e_{13}^{2} w^{3}-\gamma_{2} e_{13}^{1} w^{3}+\gamma_{3} e_{23}^{2} w^{3}-\gamma_{4} e_{23}^{1} w^{3}+\gamma_{5} e_{33}^{2} w^{3}-\gamma_{6} e_{33}^{1} w^{3}-\gamma_{5} C_{12}^{1} w^{3}-\gamma_{6} C_{12}^{2} w^{3}
\end{array}\right)=\left(\begin{array}{l}
0 \\
0 \\
0
\end{array}\right)
$$

2.6. Determination of the $\operatorname{ker} \tilde{d}^{1}$ and $\operatorname{Im} \tilde{d}^{1}$

$$
\operatorname{ker} \tilde{d}^{1}=\left\{A \in M_{3 \times 2}(\mathbb{R}) \mid \tilde{d}^{1} A=0\right\} .
$$


$\tilde{d}^{1} A=0$ iff

$$
\left(\begin{array}{l}
a_{1} e_{11}^{2}-a_{2} e_{11}^{1}+b_{1} e_{21}^{2}-b_{2} e_{21}^{1}+c_{1} e_{31}^{2}-c_{2} e_{31}^{1}-a_{1} C_{12}^{1}-a_{2} C_{12}^{2} \\
a_{1} e_{12}^{2}-a_{2} e_{12}^{1}+b_{1} e_{22}^{2}-b_{2} e_{22}^{1}+c_{1} e_{32}^{2}-c_{2} e_{32}^{1}-b_{1} C_{12}^{1}-b_{2} C_{12}^{2} \\
a_{1} e_{13}^{2}-a_{2} e_{12}^{1}+b_{1} e_{32}^{2}-b_{2} e_{32}^{1}+c_{1} e_{33}^{2}-c_{2} e_{33}^{1}-c_{1} C_{12}^{1}-c_{2} C_{12}^{2}
\end{array}\right)=\left(\begin{array}{l}
0 \\
0 \\
0
\end{array}\right)
$$

iff

$$
\begin{aligned}
& a_{1} e_{11}^{2}-a_{2} e_{11}^{1}+b_{1} e_{21}^{2}-b_{2} e_{21}^{1}+c_{1} e_{31}^{2}-c_{2} e_{31}^{1}-a_{1} C_{12}^{1}-a_{2} C_{12}^{2}=0 \\
& a_{1} e_{12}^{2}-a_{2} e_{12}^{1}+b_{1} e_{22}^{2}-b_{2} e_{22}^{1}+c_{1} e_{32}^{2}-c_{2} e_{32}^{1}-b_{1} C_{12}^{1}-b_{2} C_{12}^{2}=0 \\
& a_{1} e_{13}^{2}-a_{2} e_{12}^{1}+b_{1} e_{32}^{2}-b_{2} e_{32}^{1}+c_{1} e_{33}^{2}-c_{2} e_{33}^{1}-c_{1} C_{12}^{1}-c_{2} C_{12}^{2}=0 .
\end{aligned}
$$

Now, we compute the $\operatorname{Im} \tilde{d}^{1}$ using the standard basis

$$
A_{1}=\left(\begin{array}{ll}
1 & 0 \\
0 & 0 \\
0 & 0
\end{array}\right), \quad A_{2}=\left(\begin{array}{ll}
0 & 1 \\
0 & 0 \\
0 & 0
\end{array}\right), \quad A_{3}=\left(\begin{array}{ll}
0 & 0 \\
1 & 0 \\
0 & 0
\end{array}\right), \quad A_{4}=\left(\begin{array}{ll}
0 & 0 \\
0 & 1 \\
0 & 0
\end{array}\right), \quad A_{5}=\left(\begin{array}{ll}
0 & 0 \\
0 & 0 \\
1 & 0
\end{array}\right), \quad A_{6}=\left(\begin{array}{ll}
0 & 0 \\
0 & 0 \\
0 & 1
\end{array}\right) .
$$

If $A_{1}=\left(\begin{array}{ll}1 & 0 \\ 0 & 0 \\ 0 & 0\end{array}\right)$ then $\tilde{d}^{1} A_{1}=\left(\begin{array}{c}e_{11}^{2}-C_{12}^{2} \\ e_{12}^{2} \\ e_{13}^{2}\end{array}\right)$.

If $A_{2}=\left(\begin{array}{ll}0 & 1 \\ 0 & 0 \\ 0 & 0\end{array}\right)$ then $\tilde{d}^{1} A_{2}=\left(\begin{array}{c}-e_{11}^{1}-C_{12}^{1} \\ -e_{12}^{1} \\ -e_{13}^{1}\end{array}\right)$.

If $A_{3}=\left(\begin{array}{ll}0 & 0 \\ 1 & 0 \\ 0 & 0\end{array}\right)$ then $\tilde{d}^{1} A_{3}=\left(\begin{array}{c}e_{11}^{2} \\ e_{22}^{2}-C_{12}^{2} \\ e_{23}^{2}\end{array}\right)$.

If $A_{4}=\left(\begin{array}{ll}0 & 0 \\ 0 & 1 \\ 0 & 0\end{array}\right)$ then $\tilde{d}^{1} A_{4}=\left(\begin{array}{c}-e_{21}^{1} \\ -e_{22}^{1}-C_{12}^{1} \\ -e_{23}^{1}\end{array}\right)$.

If $A_{5}=\left(\begin{array}{ll}0 & 0 \\ 0 & 0 \\ 1 & 0\end{array}\right)$ then $\tilde{d}^{1} A_{5}=\left(\begin{array}{c}e_{31}^{2} \\ e_{32}^{2} \\ e_{33}^{2}-C_{12}^{2}\end{array}\right)$.

If $A_{6}=\left(\begin{array}{ll}0 & 0 \\ 0 & 0 \\ 0 & 1\end{array}\right)$ then $\tilde{d}^{1} A_{6}=\left(\begin{array}{c}-e_{31}^{1} \\ -e_{32}^{1} \\ -e_{33}^{1}-C_{12}^{1}\end{array}\right)$.

Thus, we have the image matrix as follows:

$$
\tilde{M}_{1}=\left(\begin{array}{cccccc}
e_{11}^{2}-C_{12}^{2} & -e_{11}^{1}-C_{12}^{1} & e_{21}^{2} & -e_{21}^{1} & e_{31}^{2} & -e_{31}^{1} \\
e_{12}^{2} & -e_{12}^{1} & e_{22}^{2}-C_{12}^{2} & -e_{22}^{1}-C_{12}^{1} & e_{32}^{2} & -e_{32}^{1} \\
e_{13}^{2} & -e_{13}^{1} & e_{23}^{2} & -e_{23}^{1} & e_{33}^{2}-C_{12}^{2} & -e_{33}^{1}-C_{12}^{1}
\end{array}\right) .
$$

Next, we calculate the rank of the matrix $\tilde{M}_{1}$ which will help us to know the $\operatorname{ker} \tilde{d}^{1}$ and $\operatorname{Im} \tilde{d}^{1}$ by using the dimension rank theorem of the vector spaces [5] [6].

We now reduce the matrix $\tilde{M}_{1}$ to reduce row echelon form. We then replace the entries of the matrix $\tilde{M}_{1}$ by the follows constants: 


$$
\left(\begin{array}{llllll}
a_{11} & a_{12} & a_{13} & a_{14} & a_{15} & a_{16} \\
a_{21} & a_{22} & a_{23} & a_{24} & a_{25} & a_{26} \\
a_{31} & a_{32} & a_{33} & a_{34} & a_{35} & a_{36}
\end{array}\right)
$$

where $\left\{\begin{array}{l}a_{11}=e_{11}^{2}-C_{12}^{2} ; \\ a_{12}=-e_{11}^{1}-C_{12}^{1} ; \\ a_{13}=e_{21}^{2} ; \\ a_{14}=-e_{21}^{1} ; \\ a_{15}=-e_{31}^{2} ; \\ a_{16}=-e_{31}^{1} .\end{array} \quad\left\{\begin{array}{l}a_{21}=e_{12}^{2} ; \\ a_{22}=-e_{12}^{1} ; \\ a_{23}=e_{22}^{2}-C_{12}^{2} ; \\ a_{24}=-e_{22}^{1}-C_{12}^{1} ; \\ a_{25}=e_{32}^{2} ; \\ a_{26}=-e_{31}^{1}\end{array} \quad\left\{\begin{array}{l}a_{31}=e_{13}^{2} ; \\ a_{31}=-e_{13}^{1} ; \\ a_{31}=e_{23}^{2} ; \\ a_{31}=-e_{23}^{1} ; \\ a_{31}=e_{33}^{2}-C_{12}^{2} ; \\ a_{31}=e_{12}^{2}-C_{12}^{2} .\end{array}\right.\right.\right.$ Let $e_{11}^{2}-C_{12}^{2} \neq 0$ and by dividing each of the

entries of row 1 by $e_{11}^{2}-C_{12}^{2}$ and carrying out the following row operation $e_{12}^{2} R_{1}-R_{2}$ and $e_{13}^{2} R_{1}-R_{3}$, we obtain

$$
A=\left(\begin{array}{cccccc}
1 & \frac{a_{12}}{a_{11}} & \frac{a_{13}}{a_{11}} & \frac{a_{14}}{a_{11}} & \frac{a_{15}}{a_{11}} & \frac{a_{16}}{a_{11}} \\
0 & a_{21}\left(\frac{a_{12}}{a_{11}}\right)-a_{22} & a_{21}\left(\frac{a_{13}}{a_{11}}\right)-a_{23} & a_{21}\left(\frac{a_{14}}{a_{11}}\right)-a_{24} & a_{21}\left(\frac{a_{15}}{a_{11}}\right)-a_{25} & a_{21}\left(\frac{a_{16}}{a_{11}}\right)-a_{26} \\
0 & a_{31}\left(\frac{a_{12}}{a_{11}}\right)-a_{32} & a_{31}\left(\frac{a_{13}}{a_{11}}\right)-a_{33} & a_{31}\left(\frac{a_{14}}{a_{11}}\right)-a_{34} & a_{31}\left(\frac{a_{15}}{a_{11}}\right)-a_{35} & a_{31}\left(\frac{a_{16}}{a_{11}}\right)-a_{36}
\end{array}\right)
$$

Let $\alpha=e_{12}^{2}\left(\frac{-e_{11}^{1}-C_{12}^{1}}{e_{11}^{2}-C_{12}^{2}}\right)-\left(e_{22}^{2}-C_{12}^{2}\right)$ be such that $\alpha \neq 0$ and by carrying the following row operations $\frac{1}{\alpha} R_{2}, \frac{-e_{11}^{1}-C_{12}^{1}}{e_{11}^{2}-C_{12}^{2}} R_{2}-R_{1}$ and $\left(e_{13}^{2}\left(\frac{\left(-e_{11}^{1}-C_{12}^{1}\right)}{\left(e_{11}^{2}-C_{12}^{2}\right)}\right)-e_{23}^{2}\right) R_{2}-R_{3}$, and setting $\left(e_{13}^{2}\left(\frac{\left(-e_{11}^{1}-C_{12}^{1}\right)}{\left(e_{11}^{2}-C_{12}^{2}\right)}\right)-\left(-e_{13}^{1}\right)\right)=t$, thus we obtain the following matrix.

$$
B=\left(\begin{array}{ccc}
1 & 0 & 0 \\
\frac{1}{\alpha} \frac{a_{12}}{a_{11}}\left(a_{21}\left(\frac{a_{13}}{a_{11}}\right)-a_{23}\right)-\frac{a_{13}}{a_{11}} & \frac{1}{\alpha}\left(a_{21}\left(\frac{a_{13}}{a_{11}}\right)-a_{23}\right) & \frac{1}{\alpha} t\left(a_{21}\left(\frac{a_{13}}{a_{11}}\right)-a_{23}\right)-\left(a_{31}\left(\frac{a_{13}}{a_{11}}\right)-a_{33}\right) \\
\frac{1}{\alpha} \frac{a_{12}}{a_{11}}\left(a_{21}\left(\frac{a_{14}}{a_{11}}\right)-a_{24}\right)-\frac{a_{14}}{a_{11}} & \frac{1}{\alpha}\left(a_{21}\left(\frac{a_{14}}{a_{11}}\right)-a_{24}\right) & \frac{1}{\alpha} t\left(a_{21}\left(\frac{a_{14}}{a_{11}}\right)-a_{24}\right)-\left(a_{31}\left(\frac{a_{14}}{a_{11}}\right)-a_{34}\right) \\
\frac{1}{\alpha} \frac{a_{12}}{a_{11}}\left(a_{21}\left(\frac{a_{15}}{a_{11}}\right)-a_{25}\right)-\frac{a_{15}}{a_{11}} & \frac{1}{\alpha}\left(a_{21}\left(\frac{a_{15}}{a_{11}}\right)-a_{25}\right) & \frac{1}{\alpha} t\left(a_{21}\left(\frac{a_{15}}{a_{11}}\right)-a_{25}\right)-\left(a_{31}\left(\frac{a_{15}}{a_{11}}\right)-a_{35}\right) \\
\frac{1}{\alpha} \frac{a_{12}}{a_{11}}\left(a_{21}\left(\frac{a_{16}}{a_{11}}\right)-a_{26}\right)-\frac{a_{16}}{a_{11}} & \frac{1}{\alpha}\left(a_{21}\left(\frac{a_{16}}{a_{11}}\right)-a_{26}\right) & \frac{1}{\alpha} t\left(a_{21}\left(\frac{a_{16}}{a_{11}}\right)-a_{26}\right)-\left(a_{31}\left(\frac{a_{16}}{a_{11}}\right)-a_{36}\right)
\end{array}\right)
$$

Let $\beta=\frac{1}{\alpha} t\left(e_{12}^{2}\left(\frac{e_{11}^{2}}{\left(e_{11}^{2}-C_{12}^{2}\right)}\right)-\left(e_{22}^{2}-C_{12}^{2}\right)\right)-\left(e_{13}^{2}\left(\frac{e_{11}^{2}}{\left(e_{11}^{2}-C_{12}^{2}\right)}\right)-\left(e_{23}^{2}\right)\right)$ be such that $\beta \neq 0$, and by carry the following row operations

$$
\frac{1}{\beta} R_{3}, \frac{1}{\beta}\left[\frac{1}{\alpha} \frac{a_{12}}{a_{11}}\left(a_{21}\left(\frac{a_{13}}{a_{11}}\right)-a_{23}\right)-\frac{a_{13}}{a_{11}}\right] R_{3}-R_{1} \text { and } \frac{1}{\beta} \frac{1}{\alpha}\left(a_{21}\left(\frac{a_{13}}{a_{11}}\right)-a_{23}\right) R_{3}-R_{2} \text {. By setting }
$$


$m=\frac{1}{\beta}\left[\frac{1}{\alpha} \frac{a_{12}}{a_{11}}\left(a_{21}\left(\frac{a_{13}}{a_{11}}\right)-a_{23}\right)-\frac{a_{13}}{a_{11}}\right]$ and $n=\frac{1}{\beta} \frac{1}{\alpha}\left(a_{21}\left(\frac{a_{13}}{a_{11}}\right)-a_{23}\right)$. Also, if we let

$$
\begin{aligned}
& x=\left[\frac{1}{\alpha} t\left(a_{21}\left(\frac{a_{14}}{a_{11}}\right)-a_{24}\right)-\left(a_{31}\left(\frac{a_{14}}{a_{11}}\right)-a_{34}\right)\right], \\
& y=\left[\frac{1}{\alpha} t\left(a_{21}\left(\frac{a_{15}}{a_{11}}\right)-a_{25}\right)-\left(a_{31}\left(\frac{a_{15}}{a_{11}}\right)-a_{35}\right)\right] \\
& z=\left[\frac{1}{\alpha} t\left(a_{21}\left(\frac{a_{16}}{a_{11}}\right)-a_{26}\right)-\left(a_{31}\left(\frac{a_{16}}{a_{11}}\right)-a_{36}\right)\right],
\end{aligned}
$$

we obtain the following matrix.

$$
\left(\begin{array}{cccc}
1 & 0 & 0 \\
0 & 1 & 0 \\
0 & 0 & 1 \\
m \frac{1}{\beta} x-\frac{1}{\alpha} \frac{a_{12}}{a_{11}}\left(a_{21}\left(\frac{a_{14}}{a_{11}}\right)-a_{24}\right)-\frac{a_{14}}{a_{11}} & n \frac{1}{\beta} x-\frac{1}{\alpha}\left(a_{21}\left(\frac{a_{14}}{a_{11}}\right)-a_{24}\right) & \frac{1}{\beta} x \\
m \frac{1}{\beta} y-\frac{1}{\alpha} \frac{a_{12}}{a_{11}}\left(a_{21}\left(\frac{a_{15}}{a_{11}}\right)-a_{25}\right)-\frac{a_{15}}{a_{11}} & n \frac{1}{\beta} y-\frac{1}{\alpha}\left(a_{21}\left(\frac{a_{15}}{a_{11}}\right)-a_{25}\right) & \frac{1}{\beta} y \\
m \frac{1}{\beta} z-\frac{1}{\alpha} \frac{a_{12}}{a_{11}}\left(a_{21}\left(\frac{a_{16}}{a_{11}}\right)-a_{26}\right)-\frac{a_{16}}{a_{11}} & n \frac{1}{\beta} z-\frac{1}{\alpha}\left(a_{21}\left(\frac{a_{16}}{a_{11}}\right)-a_{26}\right) & \frac{1}{\beta} z
\end{array}\right) .
$$

Hence we obtain the reduce row echelon form of $\tilde{M}_{1}$ of rank 3 [5] [6].

We wish to consider now the cases of the matrix $\tilde{M}_{1}$ of rank 1 and rank 2 since the case of rank Zero is trivial.

Rank 1: By setting each of the entries on row 2 and 3 of matrix $A$ to zero, we obtain the rank of $\tilde{M}_{1}$ to be 1 .

Rank 2: By setting each of the entries on row 3 of matrix $B$ to zero, we obtain the rank of $\tilde{M}_{1}$ to be 2 .

Proposition 3: if

$$
\begin{aligned}
& \left(e_{11}^{2}-C_{12}^{2}\right)=0,\left(-e_{11}^{1}-C_{12}^{1}\right)=0, e_{11}^{2}=0,-e_{21}^{1}=0, e_{31}^{2}=0,-e_{31}^{1}=0, \\
& e_{12}^{2}=0,-e_{12}^{1}=0,\left(e_{22}^{2}-C_{12}^{2}\right)=0,\left(-e_{22}^{1}-C_{12}^{1}\right)=0, e_{32}^{2}=0,-e_{32}^{1}=0, \\
& e_{13}^{2}=0,-e_{13}^{1}=0, e_{23}^{2}=0,-e_{23}^{1}=0, e_{33}^{2}-C_{12}^{2}=0,-e_{33}^{1}-C_{12}^{1}=0,
\end{aligned}
$$

then $\left(\begin{array}{llllll}0 & 0 & 0 & 0 & 0 & 0 \\ 0 & 0 & 0 & 0 & 0 & 0 \\ 0 & 0 & 0 & 0 & 0 & 0\end{array}\right)$ and the $\operatorname{rank} \tilde{M}_{1}=0$. Further

$\operatorname{ker} \tilde{d}^{1} \cong \mathbb{R}^{6}$ and $\operatorname{Im} \tilde{d}^{1} \cong 0$.

Proposition 4: From matrix A, if $e_{11}^{2}-C_{12}^{2} \neq 0,\left(e_{11}^{2}-C_{12}^{2}\right)\left(\frac{\left(-e_{11}^{1}-C_{12}^{1}\right)}{\left(e_{11}^{2}-C_{12}^{2}\right)}\right)-\left(-e_{12}^{1}\right)=0$,

$e_{12}^{2}\left(\frac{e_{11}^{2}}{\left(e_{11}^{2}-C_{12}^{2}\right)}\right)-\left(e_{22}^{2}-C_{12}^{2}\right)=0, \quad e_{12}^{2}\left(\frac{\left(-e_{21}^{1}\right)}{\left(e_{11}^{2}-C_{12}^{2}\right)}\right)-\left(-e_{22}^{1}-C_{12}^{1}\right)=0, \quad e_{12}^{2}\left(\frac{e_{31}^{2}}{\left(e_{11}^{2}-C_{12}^{2}\right)}\right)-e_{32}^{2}=0$, 
$e_{12}^{2}\left(\frac{\left(-e_{31}^{1}\right)}{\left(e_{11}^{2}-C_{12}^{2}\right)}\right)-\left(-e_{32}^{1}\right)=0$, then

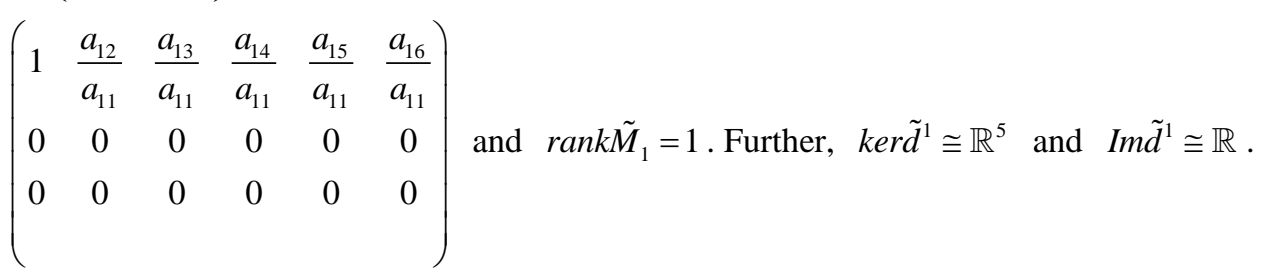

Proof. Since the $\operatorname{rank} \tilde{M}_{1}=1$, we have that $\operatorname{dim}\left(\operatorname{Im} \tilde{d}^{1}\right)=1$, thus $\operatorname{Im} \tilde{d}^{1} \cong \mathbb{R}$. We now show that $\operatorname{ker\tilde {d}^{1}} \cong \mathbb{R}^{5}$. By the dimension rank theorem, we have that $\operatorname{dim}\left(\operatorname{ker\tilde {d}^{1}}\right)+\operatorname{dim}\left(\operatorname{Im} \tilde{\tilde{d}}^{1}\right)=6$ which is $\operatorname{dim}\left(\operatorname{ker} \tilde{d}^{1}\right)=6-1=5$.

Proposition 5: From matrix $B$, if $e_{12}^{2}\left(\frac{\left(-e_{11}^{1}-C_{12}^{1}\right)}{\left(e_{11}^{2}-C_{12}^{2}\right)}\right)-\left(-e_{12}^{1}\right) \neq 0$,

$\frac{1}{\alpha} t\left(e_{12}^{2}\left(\frac{e_{11}^{2}}{\left(e_{11}^{2}-C_{12}^{2}\right)}\right)-\left(e_{22}^{2}-C_{12}^{2}\right)\right)-\left(e_{13}^{2}\left(\frac{e_{11}^{2}}{\left(e_{11}^{2}-C_{12}^{2}\right)}\right)-e_{23}^{2}\right)=0$,

$\frac{1}{\alpha} t\left(e_{12}^{2}\left(\frac{-e_{21}^{1}}{\left(e_{11}^{2}-C_{12}^{2}\right)}\right)-\left(-e_{22}^{1}-C_{12}^{1}\right)\right)-\left(e_{13}^{2}\left(\frac{-e_{21}^{1}}{\left(e_{11}^{2}-C_{12}^{2}\right)}\right)-\left(-e_{23}^{1}\right)\right)=0$,

$\frac{1}{\alpha} t\left(e_{12}^{2}\left(\frac{e_{31}^{2}}{\left(e_{11}^{2}-C_{12}^{2}\right)}\right)-e_{32}^{2}\right)-\left(e_{13}^{2}\left(\frac{e_{31}^{2}}{\left(e_{11}^{2}-C_{12}^{2}\right)}\right)-\left(e_{33}^{2}-C_{12}^{2}\right)\right)=0$,

$\frac{1}{\alpha} t\left(e_{12}^{2}\left(\frac{-e_{31}^{1}}{\left(e_{11}^{2}-C_{12}^{2}\right)}\right)-\left(-e_{32}^{1}\right)\right)-\left(e_{13}^{2}\left(\frac{-e_{31}^{1}}{\left(e_{11}^{2}-C_{12}^{2}\right)}\right)-\left(-e_{33}^{1}-C_{12}^{1}\right)\right)=0$, then

$\left(\begin{array}{ccc}1 & 0 & 0 \\ \frac{1}{\alpha} \frac{a_{12}}{a_{11}}\left(a_{21}\left(\frac{a_{13}}{a_{11}}\right)-a_{23}\right)-\frac{a_{13}}{a_{11}} & \frac{1}{\alpha}\left(a_{21}\left(\frac{a_{13}}{a_{11}}\right)-a_{23}\right) & 0 \\ \frac{1}{\alpha} \frac{a_{12}}{a_{11}}\left(a_{21}\left(\frac{a_{14}}{a_{11}}\right)-a_{24}\right)-\frac{a_{14}}{a_{11}} & \frac{1}{\alpha}\left(a_{21}\left(\frac{a_{14}}{a_{11}}\right)-a_{24}\right) & 0 \\ \frac{1}{\alpha} \frac{a_{12}}{a_{11}}\left(a_{21}\left(\frac{a_{15}}{a_{11}}\right)-a_{25}\right)-\frac{a_{15}}{a_{11}} & \frac{1}{\alpha}\left(a_{21}\left(\frac{a_{15}}{a_{11}}\right)-a_{25}\right) & 0 \\ \frac{1}{\alpha} \frac{a_{12}}{a_{11}}\left(a_{21}\left(\frac{a_{16}}{a_{11}}\right)-a_{26}\right)-\frac{a_{16}}{a_{11}} & \frac{1}{\alpha}\left(a_{21}\left(\frac{a_{16}}{a_{11}}\right)-a_{26}\right) & 0\end{array}\right)$

and $\operatorname{rank} \tilde{M}_{1}=2$. Further, $\operatorname{ker} \tilde{d}^{1} \cong \mathbb{R}^{4}$ and $\operatorname{Im} \tilde{d}^{1} \cong \mathbb{R}^{2}$.

Proof. Since the $\operatorname{rank} \tilde{M}_{1}=2$, we have that $\operatorname{dim}\left(\operatorname{Im} \tilde{d}^{1}\right)=2$, thus $\operatorname{Im} \tilde{d}^{1} \cong \mathbb{R}^{2}$. We now show that $k e r \tilde{d}^{1} \cong \mathbb{R}^{4}$. By the dimension rank theorem, we have that $\operatorname{dim}\left(\operatorname{ker} \tilde{d}^{1}\right)+\operatorname{dim}\left(\operatorname{Im} \tilde{d}^{1}\right)=6$, that is $\operatorname{dim}\left(\operatorname{ker} \tilde{d}^{1}\right)=6-2=4$.

Proposition 6: if

$e_{11}^{2}-C_{12}^{2} \neq 0, \quad \alpha=e_{12}^{2}\left(\frac{\left(-e_{11}^{1}-C_{12}^{1}\right)}{\left(e_{11}^{2}-C_{12}^{2}\right)}\right)-\left(-e_{11}^{1}-C_{12}^{1}\right) \neq 0$, 
$\beta=\frac{1}{\alpha} t\left(e_{12}^{2}\left(\frac{e_{11}^{2}}{\left(e_{11}^{2}-C_{12}^{2}\right)}\right)-\left(e_{22}^{2}-C_{12}^{2}\right)\right)-\left(e_{13}^{2}\left(\frac{a_{13}}{\left(e_{11}^{2}-C_{12}^{2}\right)}\right)-e_{23}^{2}\right) \neq 0$, then $\left(\begin{array}{cccc}1 & 0 & 0 \\ 0 & 1 & 0 \\ 0 & n \frac{1}{\beta} x-\frac{1}{\alpha} \frac{a_{12}}{a_{11}}\left(a_{21}\left(\frac{a_{14}}{a_{11}}\right)-a_{24}\right)-\frac{a_{14}}{a_{11}} & n \frac{1}{\beta}\left(a_{21}\left(\frac{a_{14}}{a_{11}}\right)-a_{24}\right) & \frac{1}{\beta} x \\ m \frac{1}{\beta} y-\frac{1}{\alpha} \frac{a_{12}}{a_{11}}\left(a_{21}\left(\frac{a_{15}}{a_{11}}\right)-a_{25}\right)-\frac{a_{15}}{a_{11}} & n \frac{1}{\beta} y-\frac{1}{\alpha}\left(a_{21}\left(\frac{a_{15}}{a_{11}}\right)-a_{25}\right) & \frac{1}{\beta} y \\ m \frac{1}{\beta} z-\frac{1}{\alpha} \frac{a_{12}}{a_{11}}\left(a_{21}\left(\frac{a_{16}}{a_{11}}\right)-a_{26}\right)-\frac{a_{16}}{a_{11}} & n \frac{1}{\beta} z-\frac{1}{\alpha}\left(a_{21}\left(\frac{a_{16}}{a_{11}}\right)-a_{26}\right) & \frac{1}{\beta} z\end{array}\right)$

and the $\operatorname{rank} \tilde{M}_{1}=0$. Further, $\operatorname{ker} \tilde{d}^{1} \cong \mathbb{R}^{3}$ and $\operatorname{Im} \tilde{d}^{1} \cong \mathbb{R}^{3}$.

Proof. Since the $\operatorname{rank} \tilde{M}_{1}=3$, we have that $\operatorname{dim}\left(\operatorname{Im} \tilde{d}^{1}\right)=3$, thus $\operatorname{Im} \tilde{d}^{1} \cong \mathbb{R}^{3}$. We now show that $\operatorname{kerd} \tilde{d}^{1} \cong \mathbb{R}^{3}$. By the dimension rank theorem, we have that $\operatorname{dim}\left(\operatorname{ker} \tilde{d}^{1}\right)+\operatorname{dim}\left(\operatorname{Im} \tilde{d}^{1}\right)=6$, that is $\operatorname{dim}\left(\operatorname{ker} \tilde{d}^{1}\right)=6-3=3$.

Now, we compute our quotient spaces of the 2 - 3 matrix Chevalley Eilenberg cohomology which are $H^{0}(V)=\operatorname{Ker} \tilde{d}^{0} / \operatorname{Im} \tilde{d}^{-1}, H^{1}(V)=\operatorname{Ker} \tilde{d}^{1} / \operatorname{Im} \tilde{d}^{0}$ and $H^{2}(V)=\operatorname{Ker} \tilde{d}^{2} / \operatorname{Im} \tilde{d}^{1}$.

For $H^{0}(V)$, we have the following quotient space:

Case 1: $\operatorname{Im}^{-1} \cong 0$ and $\operatorname{Ker}^{0} \cong 0$

$$
\operatorname{Ker} \tilde{d}^{0} / \operatorname{Im} \tilde{d}^{-1} \cong 0 .
$$

For $H^{1}(V)$, we have the following quotient spaces:

Case 1: $\operatorname{Im} \tilde{d}^{0} \cong \mathbb{R}^{0}$ and $\operatorname{Ker} \tilde{d}^{1} \cong \mathbb{R}^{6}$

$$
\operatorname{Ker} \tilde{d}^{1} / \operatorname{Im} \tilde{d}^{0} \cong \mathbb{R}^{6}
$$

Case 2: $\quad \operatorname{Im} \tilde{d}^{0} \cong \mathbb{R}$ and $\operatorname{Ker} \tilde{d}^{1} \cong \mathbb{R}^{6}$

$$
\operatorname{Ker} \tilde{d}^{1} / \operatorname{Im} \tilde{d}^{0} \cong \mathbb{R}^{5}
$$

Case 3: $\operatorname{Im} \tilde{d}^{0} \cong \mathbb{R}^{2}$ and $\operatorname{Ker} \tilde{d}^{1} \cong \mathbb{R}^{6}$

$\operatorname{Ker} \tilde{d}^{1} / \operatorname{Im} \tilde{d}^{0} \cong \mathbb{R}^{4}$.

Case 4: $\quad \operatorname{Im} \tilde{d}^{0} \cong \mathbb{R}^{3}$ and $\operatorname{Ker} \tilde{d}^{1} \cong \mathbb{R}^{6}$

$$
\operatorname{Ker} \tilde{d}^{1} / \operatorname{Im} \tilde{d}^{0} \cong \mathbb{R}^{3}
$$

Case 5: $\operatorname{Im} \tilde{d}^{0} \cong \mathbb{R}^{4}$ and $\operatorname{Ker} \tilde{d}^{1} \cong \mathbb{R}^{6}$

$$
\operatorname{Ker} \tilde{d}^{1} / \operatorname{Im} \tilde{d}^{0} \cong \mathbb{R}^{2}
$$

Case 6: $\operatorname{Im} \tilde{d}^{0} \cong \mathbb{R}^{5}$ and $\operatorname{Ker} \tilde{d}^{1} \cong \mathbb{R}^{6}$

$$
\operatorname{Ker} \tilde{d}^{1} / \operatorname{Im} \tilde{d}^{0} \cong \mathbb{R}
$$

Case 7: $\operatorname{Im} \tilde{d}^{0} \cong \mathbb{R}^{6}$ and $\operatorname{Ker} \tilde{d}^{1} \cong \mathbb{R}^{6}$

$$
\operatorname{Ker} \tilde{d}^{1} / \operatorname{Im} \tilde{d}^{0} \cong 0 .
$$

Case 1: $\operatorname{Im} \tilde{d}^{0} \cong 0$ and $\operatorname{Ker} \tilde{d}^{1} \cong \mathbb{R}^{5}$

$$
\operatorname{Ker} \tilde{d}^{1} / \operatorname{Im} \tilde{d}^{0} \cong \mathbb{R}^{5} .
$$

Case 2: $\operatorname{Im} \tilde{d}^{0} \cong \mathbb{R}$ and $\operatorname{Ker} \tilde{d}^{1} \cong \mathbb{R}^{5}$

$$
\operatorname{Ker} \tilde{d}^{1} / \operatorname{Im} \tilde{d}^{0} \cong \mathbb{R}^{4}
$$


Case 3: $\operatorname{Im} \tilde{d}^{0} \cong \mathbb{R}^{2}$ and $\operatorname{Ker} \tilde{d}^{1} \cong \mathbb{R}^{5}$

$$
\operatorname{Ker} \tilde{d}^{1} / \operatorname{Im} \tilde{d}^{0} \cong \mathbb{R}^{3} .
$$

Case 4: $\operatorname{Im} \tilde{d}^{0} \cong \mathbb{R}^{3}$ and $\operatorname{Ker} \tilde{d}^{1} \cong \mathbb{R}^{5}$

$$
\operatorname{Ker} \tilde{d}^{1} / \operatorname{Im} \tilde{d}^{0} \cong \mathbb{R}^{2}
$$

Case 5: $\operatorname{Im} \tilde{d}^{0} \cong \mathbb{R}^{4}$ and $\operatorname{Ker} \tilde{d}^{1} \cong \mathbb{R}^{5}$

$$
\operatorname{Ker} \tilde{d}^{1} / \operatorname{Im} \tilde{d}^{0} \cong \mathbb{R} .
$$

Case 6: $\operatorname{Im} \tilde{d}^{0} \cong \mathbb{R}^{5}$ and $\operatorname{Ker} \tilde{d}^{1} \cong \mathbb{R}^{5}$

$\operatorname{Ker} \tilde{d}^{1} / \operatorname{Im} \tilde{d}^{0} \cong 0$.

Case 1: $\quad \operatorname{Im} \tilde{d}^{0} \cong 0$ and $\operatorname{Ker} \tilde{d}^{1} \cong \mathbb{R}^{4}$

$$
\operatorname{Ker} \tilde{d}^{1} / \operatorname{Im} \tilde{d}^{0} \cong \mathbb{R}^{4} .
$$

Case 2: $\quad \operatorname{Im} \tilde{d}^{0} \cong \mathbb{R}$ and $\operatorname{Ker}^{1} \cong \mathbb{R}^{4}$

$$
\operatorname{Ker} \tilde{d}^{1} / \operatorname{Im} \tilde{d}^{0} \cong \mathbb{R}^{3}
$$

Case 3: $\operatorname{Im} \tilde{d}^{0} \cong \mathbb{R}^{2}$ and $\operatorname{Ker} \tilde{d}^{1} \cong \mathbb{R}^{4}$

$$
\operatorname{Ker} \tilde{d}^{1} / \operatorname{Im} \tilde{d}^{0} \cong \mathbb{R}^{2}
$$

Case 4: $\quad \operatorname{Im} \tilde{d}^{0} \cong \mathbb{R}^{3}$ and $\operatorname{Ker} \tilde{d}^{1} \cong \mathbb{R}^{4}$

$$
\operatorname{Ker} \tilde{d}^{1} / \operatorname{Im} \tilde{d}^{0} \cong \mathbb{R} .
$$

Case 5: $\quad \operatorname{Im} \tilde{d}^{0} \cong \mathbb{R}^{4}$ and $\operatorname{Ker}^{1} \cong \mathbb{R}^{4}$

$$
\operatorname{Ker} \tilde{d}^{1} / \operatorname{Im} \tilde{d}^{0} \cong 0 .
$$

Case 1: $\operatorname{Im} \tilde{d}^{0} \cong 0$ and $\operatorname{Ker} \tilde{d}^{1} \cong \mathbb{R}^{3}$

$$
\operatorname{Ker} \tilde{d}^{1} / \operatorname{Im} \tilde{d}^{0} \cong \mathbb{R}^{3}
$$

Case 2: $\quad \operatorname{Im} \tilde{d}^{0} \cong \mathbb{R}$ and $\operatorname{Ker}^{1} \cong \mathbb{R}^{3}$

$$
\operatorname{Ker} \tilde{d}^{1} / \operatorname{Im} \tilde{d}^{0} \cong \mathbb{R}^{2} .
$$

Case 3: $\quad \operatorname{Im} \tilde{d}^{0} \cong \mathbb{R}^{2}$ and $\operatorname{Ker} \tilde{d}^{1} \cong \mathbb{R}^{3}$

$$
\operatorname{Ker} \tilde{d}^{1} / \operatorname{Im} \tilde{d}^{0} \cong \mathbb{R} .
$$

Case 4: $\quad \operatorname{Im} \tilde{d}^{0} \cong \mathbb{R}^{3}$ and $\operatorname{Ker}^{1} \cong \mathbb{R}^{3}$

$$
\operatorname{Ker} \tilde{d}^{1} / \operatorname{Im} \tilde{d}^{0} \cong 0 .
$$

Case 1: $\operatorname{Im} \tilde{d}^{0} \cong 0$ and $\operatorname{Ker} \tilde{d}^{1} \cong \mathbb{R}^{2}$

$$
\operatorname{Ker} \tilde{d}^{1} / \operatorname{Im} \tilde{d}^{0} \cong \mathbb{R}^{2} .
$$

Case 2: $\operatorname{Im} \tilde{d}^{0} \cong \mathbb{R}$ and $\operatorname{Ker} \tilde{d}^{1} \cong \mathbb{R}^{2}$

$$
\operatorname{Ker} \tilde{d}^{1} / \operatorname{Im} \tilde{d}^{0} \cong \mathbb{R} .
$$

Case 3: $\operatorname{Im} \tilde{d}^{0} \cong \mathbb{R}^{2}$ and $\operatorname{Ker} \tilde{d}^{1} \cong \mathbb{R}^{2}$

$$
\operatorname{Kerd} \tilde{d}^{1} / \operatorname{Im} \tilde{d}^{0} \cong 0 .
$$

Case 1: $\operatorname{Im} \tilde{d}^{0} \cong 0$ and $\operatorname{Ker}^{1} \cong \mathbb{R}$

$$
\operatorname{Ker} \tilde{d}^{1} / \operatorname{Im} \tilde{d}^{0} \cong \mathbb{R} .
$$


Case 2: $\quad \operatorname{Im} \tilde{d}^{0} \cong \mathbb{R}$ and $\operatorname{Ker} \tilde{d}^{1} \cong \mathbb{R}$

$$
\operatorname{Ker} \tilde{d}^{1} / \operatorname{Im} \tilde{d}^{0} \cong 0 .
$$

For $H^{2}(V)$, we have the following quotient spaces:

Case 1: $\operatorname{Im} \tilde{d}^{1} \cong 0$ and $\operatorname{Ker}^{2} \cong \mathbb{R}^{3}$

$$
\operatorname{Ker} \tilde{d}^{2} / \operatorname{Im} \tilde{d}^{1} \cong \mathbb{R}^{3}
$$

Case 2: $\quad \operatorname{Im} \tilde{d}^{1} \cong \mathbb{R}$ and $\operatorname{Ker} \tilde{d}^{2} \cong \mathbb{R}^{3}$

$$
\operatorname{Ker} \tilde{d}^{2} / \operatorname{Im} \tilde{d}^{1} \cong \mathbb{R}^{2}
$$

Case 3: $\operatorname{Im} \tilde{d}^{1} \cong \mathbb{R}^{2}$ and $\operatorname{Kerd} \tilde{d}^{2} \cong \mathbb{R}^{3}$

$$
\operatorname{Ker} \tilde{d}^{2} / \operatorname{Im} \tilde{d}^{1} \cong \mathbb{R}
$$

Case 4: $\quad \operatorname{Im} \tilde{d}^{1} \cong \mathbb{R}^{3}$ and $\operatorname{Kerd} \tilde{d}^{2} \cong \mathbb{R}^{3}$

$$
\operatorname{Ker} \tilde{d}^{2} / \operatorname{Im} \tilde{d}^{1} \cong 0 .
$$

We suggest that further research in this direction is to carry out the deformation on the Cohomological spaces $H^{0}, H^{1}$ and $H^{2}$ which are 32 in number and apply a specific example with $\mathfrak{s l}_{2}(\mathbb{C})$. We will also carry out an extensive study on the solution of our system of linear equations on the $2-3$ matrix Chavelley Eilenberg fundamental condition.

\section{Acknowledgements}

We thank the Editor and the referee for their comments.

\section{References}

[1] Chevalley, C. and Eilenberg, S. (1948) Cohomology Theory of Lie Groups and Lie Algebras. Transactions of the American Mathematical Society, 63, 85-124. http://dx.doi.org/10.1090/S0002-9947-1948-0024908-8

[2] Nijenhuis, A. and Richardson, R.W. (1967) Deformation of Lie Algebra Structures. Journal of Mathematics and Mechanics, 17, No. 1.

[3] Hochschild, G., Kostant, B. and Rosenberg, A. (1962) Differential Forms On Regular Affine Algebras. Transactions of the American Mathematical Society, 102, 383-408. http://dx.doi.org/10.1090/S0002-9947-1962-0142598-8

[4] Goze, M. (1986) Perturbations of Lie Algebra Structures. In: Hazewinkel, M. and Gerstenhaber, M., Eds., Deformation Theory of Lie Algebra and Structures and Application, NATO ASI Series, Vol. 247, Springer, Netherlands, 265-355.

[5] Hefferon, J. (2001) Linear Algebra. Saint Michaels College Colchester, Vermont.

[6] Giarlet, P. (1998) Introduction à l'analyse numrique matricielle et l'optimisation. DUMOD. 